\title{
Dynamics of almost periodic Schoener's competition model with time delays and impulses
}

\author{
Can Li, Zunguang Guo and Zhiyu Zhang*
}

\section{*Correspondence:} zhangzhiyu008@aliyun.com Department of Science, Taiyuan Institute of Technology, Taiyuan 030008, Shanxi, China

\begin{abstract}
In real world, the ecological systems are usually perturbed by human exploitation activities such as planting and harvesting and so on. In order to obtain a more accurate description for such phenomenon, the impulsive differential equations play an important role. This paper is concerned with a kind of almost periodic Schoener's competition model with pure-delays and impulsive effects. By using the comparison theorem and the Lyapunov functional method of the impulsive differential equations, some sufficient conditions are obtained for the persistence and existence of a unique uniformly asymptotically stable positive almost periodic solution in a class of impulsive Schoener's competition model with pure-delays. The main results in this paper improve some results in recent years. And the method used in this paper provides a possible and effective method to study the permanence and existence of a unique uniformly asymptotically stable positive almost periodic solution of the models with impulsive perturbations in biological populations. Finally, an example and numerical simulations are given to illustrate the feasibility and effectiveness of our main results.
\end{abstract}

Keywords: Comparison theorem, Lyapunov functional, Persistence, Almost periodicity, Impulsive differential equations

Mathematics Subject Classification: 34K14, 34K20, 34K45, 92D25

\section{Background}

One of the most interest topics in mathematics ecology concerns the uniform persistence, almost periodic oscillations and global stability of ecology system. It is well known that a lot of Lotka-Volterra competitive systems have been discussed. Chen (1988) proposed and discussed a more practical competitive model as follows:

$$
\left\{\begin{array}{l}
\dot{x}_{1}(t)=x_{1}(t)\left[\frac{a_{10}(t)}{x_{1}(t)+m_{1}(t)}-a_{11}(t) x_{1}(t)-a_{12}(t) x_{2}(t)-c_{1}(t)\right], \\
\dot{x}_{2}(t)=x_{2}(t)\left[\frac{a_{20}(t)}{x_{2}(t)+m_{2}(t)}-a_{21}(t) x_{1}(t)-a_{22}(t) x_{2}(t)-c_{2}(t)\right] .
\end{array}\right.
$$

In biological terms, $x_{1}$ and $x_{2}$ stand for the spatial densities of each species and $c_{1}$ and $c_{2}$ are their respective net death rate. The coefficients $a_{11}$ and $a_{22}$ are intra-specific

\section{Springer Open}

(c) $2016 \mathrm{Li}$ et al. This article is distributed under the terms of the Creative Commons Attribution 4.0 International License (http:// creativecommons.org/licenses/by/4.0/), which permits unrestricted use, distribution, and reproduction in any medium, provided you give appropriate credit to the original author(s) and the source, provide a link to the Creative Commons license, and indicate if changes were made. 
competition rates whereas $a_{12}$ and $a_{21}$ are inter-specific competition rates. The asymptotic behaviors of the solution to the system (1) have been studied in Liu et al. (2006).

It is well known that the assumption of almost periodicity of the coefficients in systems is a way of incorporating the time-dependent variability of the environment, especially when the various components of the environment are periodic with not necessary commensurate periods (e.g., climate change, food supplies, mating habits and harvesting). For this reason, the assumption of almost periodicity is more realistic, more important and more general when we consider the effects of the environmental factors. In recent years, there are many scholars concerning with the Schoener's competition system. Topics such as existence, uniqueness and global attractivity of positive periodic solutions or almost periodic solutions of the system were extensively investigated, and many excellent results have been derived (see Xue et al. 2015; Tian et al. 2015; Zhang et al. 2015; Liu and Xu 2004; Liu et al. 2006; Li and Yang 2009; Gan and Lin 2012; Wu et al. 2009; Zhang et al. 2012 and the references cited therein).

On the other hand, many evolution processes are characterized by the fact that at certain moments of time they experience a change of state abruptly. These processes are subject to short-term perturbations whose duration is negligible in comparison with the duration of the process. Consequently, it is natural to assume that these perturbations act instantaneously, that is, in the form of impulses. Thus impulsive differential equations, that is, differential equations involving impulse effects, appear as a natural description of observed evolution phenomena of real world problems. The ecological systems are often deeply perturbed by human exploitation activities such as planting and harvesting and so on, which makes them unsuitable to be considered continually. In recent years, the impulsive problems in ecological systems have been intensively investigated (see Lakshmikantham et al. 1989; Stamov 2012; Samoilenko and Perestyuk 1995; Bainov and Simeonov 1993; Jin et al. 2005; Stamov 2009; Liu and Chen 2007; He et al. 2010; Zhang et al. 2014; Zhang and Li 2011 for more detail). For instance, Zhang et al. (2012) studied the following almost periodic Schoener's competition model with pure-delays and impulsive effects:

$$
\left\{\begin{array}{l}
\dot{x}_{1}(t)=x_{1}(t)\left[\frac{a_{10}(t)}{x_{1}\left(t-\tau_{10}\right)+m_{1}(t)}-a_{11}(t) x_{1}\left(t-\tau_{11}\right)-a_{12}(t) x_{2}\left(t-\tau_{12}\right)-c_{1}(t)\right] \\
\dot{x}_{2}(t)=x_{2}(t)\left[\frac{a_{20}(t)}{x_{2}\left(t-\tau_{20}\right)+m_{2}(t)}-a_{21}(t) x_{1}\left(t-\tau_{21}\right)-a_{22}(t) x_{2}\left(t-\tau_{22}\right)-c_{2}(t)\right], \quad t \neq \tau_{k} \\
\Delta x_{1}\left(\tau_{k}\right)=h_{1 k} x_{1}\left(\tau_{k}\right), \\
\Delta x_{2}\left(\tau_{k}\right)=h_{2 k} x_{2}\left(\tau_{k}\right), \quad k \in \mathbb{Z}^{+}:=\{0,1, \ldots\}
\end{array}\right.
$$

where $x_{1}(t), x_{2}(t)$ are population densities of species $x_{1}, x_{2}$ at time $t$, respectively, $\tau_{i j}$ are all nonnegative integers, $a_{i j}, m_{i}$ and $c_{i}$, are all positive almost periodic functions, $i=1,2, j=0,1,2, h_{1 k}, h_{2 k} \geq 0$ are almost periodic sequences, $0=\tau_{0}<\tau_{1}<\tau_{2}<\cdots<\tau_{k}<\tau_{k+1}<\cdots$, are impulse points with $\lim _{k \rightarrow+\infty} \tau_{k}=+\infty$, and the set of sequences $\left\{\tau_{k}^{j}\right\}, \tau_{k}^{j}=\tau_{k+j}-\tau_{k}, k \in \mathbb{Z}^{+}, j \in \mathbb{Z}$ is uniformly almost periodic (see Definition 1 in "Preliminaries" section).

The permanence and almost periodicity of all species in multispecies community are very important in biological populations. In recent years, the permanence and almost 
periodic solution of the models in biological populations has been studied by many authors (see Zhang 2013, 2014; Du and Lv 2013; Meng and Chen 2006; Lin and Chen 2009; Zhou et al. 2013; Zhang et al. 2014; Xia et al. 2008; Xia 2013; Liao and Zhang 2012; Liao and $\mathrm{Xu} 2014$ and the references cited therein). In these cases, the comparison theorems and the Liapunov functional method of differential equations have been applied to obtain the permanence and almost periodic solutions. However, we find few papers referring to the discontinuous systems (i.e., impulsive systems).

Recently, there are some scholars concerning with the permanence and almost periodic solution of the impulsive models in biological populations, see Zhang et al. (2012, 2014), He et al. (2010). For example, He et al. (2010) considered the following impulsive differential equation model of plankton allelopathy

$$
\left\{\begin{array}{l}
\dot{x}_{1}(t)=x_{1}(t)\left[r_{1}(t)-a_{11}(t) x_{1}(t)-a_{12}(t) x_{1}(t)-b_{1}(t) x_{1}(t) x_{2}(t)\right], \\
\dot{x}_{2}(t)=x_{2}(t)\left[r_{1}(t)-a_{21}(t) x_{1}(t)-a_{22}(t) x_{2}(t)-b_{2}(t) x_{1}(t) x_{2}(t)\right], \quad t \neq t_{k}, \\
\Delta x_{1}\left(t_{k}\right)=h_{1 k} x_{1}\left(t_{k}\right), \\
\Delta x_{2}\left(t_{k}\right)=h_{2 k} x_{2}\left(t_{k}\right), \quad k \in \mathbb{Z}^{+} .
\end{array}\right.
$$

By using the relation between the solutions of impulsive system and the corresponding non-impulsive system, the authors transformed impulsive system (3) into a continuous system. Further, by means of the comparison theorems and the Liapunov functional method of differential equations without impulses, the authors obtained some sufficient conditions ensuring the existence of a unique uniformly asymptotically stable positive almost periodic solution of system (3).

Next, by using a similar method as that in He et al. (2010), the authors in Zhang et al. (2012, 2014) studied the permanence and almost periodic solution of system (2) and the following impulsive multispecies mutualism system:

$$
\left\{\begin{array}{l}
\dot{x}_{i}(t)=x_{i}(t)\left[r_{i}(t)-a_{i}(t) x_{i}\left(t-\tau_{i}(t)\right)+\sum_{j=1, j \neq i}^{n} b_{i j}(t) \frac{x_{j}(t)}{1+x_{j}(t)}\right], \quad t \neq t_{k}, \\
\Delta x_{i}\left(t_{k}\right)=h_{i k} x_{i}\left(t_{k}\right), \quad k \in \mathbb{Z}^{+}, \quad i=1,2, \ldots, n .
\end{array}\right.
$$

It is worthwhile to note that the main results of paper (Zhang et al. 2012, 2014; He et al. 2010) indicates the impulsive coefficients $h_{i k}$ in system (2)-(4) satisfying the following condition:

(F) $H_{i}(t):=\prod_{0<t_{k}<t}\left(1+h_{i k}\right), i=1,2$ or $i=1,2, \ldots, n$ (for system (4)), $k \in \mathbb{Z}^{+}$is almost periodic function and there exist positive constants $H_{i}^{u}$ and $H_{i}^{l}$ such that $H_{i}^{l} \leq H_{i}(t) \leq H_{i}^{u}$.

Remark 1 Obviously, condition $(F)$ is too harsh. For example, if the impulse coefficient $h_{i k} \equiv 0.3(i=1,2)$ in system (2)-(4), then $(F)$ is invalid. Therefore, the main results in papers (Zhang et al. 2012, 2014; He et al. 2010) are difficult to apply to more extensive model with impulsive effects. 
In Zhang et al. (2012, 2014), He et al. (2010), although the authors considered the impulsive system, but still used the research method of continuous systems. Stimulated by this, by means of the comparison theorem and the Lyapunov functional method of the impulsive differential equations (Lakshmikantham et al. 1989; Stamov 2012), the main purpose of this paper is to establish some sufficient conditions which guarantee the permanence and existence of a unique uniformly asymptotically stable positive almost periodic solution of system (2). The main results obtained in this paper remove the harsh condition $(F)$ and provide a possible and effective method to study the permanence and existence of a unique uniformly asymptotically stable positive almost periodic solution of the models with impulsive perturbations in biological populations.

Let $\mathbb{R}$ and $\mathbb{Z}$ denote the sets of real numbers and integers integers, respectively. Related to a continuous function $f$, we use the following notations:

$$
f^{l}=\inf _{s \in \mathbb{R}} f(s), \quad f^{u}=\sup _{s \in \mathbb{R}} f(s) .
$$

The organization of this paper is as follows. In "Preliminaries" section, we give some basic definitions and necessary lemmas which will be used in later sections. In "Permanence" section, by using the comparison theorem of the impulsive differential equations (Lakshmikantham et al. 1989), we give the permanence of system (2). In "Almost periodic solution" section, we study the existence of a unique uniformly asymptotically stable positive almost periodic solution of system (2) by applying the Lyapunov method of the impulsive differential equations (Stamov 2012). Finally, an example and numerical simulations are given to illustrate that our results are feasible.

\section{Preliminaries}

Now, let us state the following definitions and lemmas, which will be useful in proving our main result.

By $\mathbb{I}, \quad \mathbb{I}=\left\{\left\{\tau_{k}\right\} \in \mathbb{R}: \tau_{k}<\tau_{k+1}, \quad k \in \mathbb{Z}, \lim _{k \rightarrow \pm \infty} \tau_{k}= \pm \infty\right\}$, we denote the set of all sequences that are unbounded and strictly increasing. Let $\Omega \subset \mathbb{R}, \Omega \neq \emptyset$, $\tau=\max \left\{2 \tau_{i j}, i=1,2, j=0,1,2\right\}, \xi_{0} \in \mathbb{R}$, introduce the following notations:

$P C\left(\xi_{0}\right)$ is the space of all functions $\phi:\left[\xi_{0}-\tau, \xi_{0}\right] \rightarrow \Omega$ having points of discontinuity at $\mu_{1}, \mu_{2}, \ldots \in\left[\xi_{0}-\tau, \xi_{0}\right]$ of the first kind and left continuous at these points.

For $J \subset \mathbb{R}, P C(J, \mathbb{R})$ is the space of all piecewise continuous functions from $J$ to $\mathbb{R}$ with points of discontinuity of the first kind $\tau_{k}$, at which it is left continuous.

Let $\phi_{1}, \phi_{2} \in P C(0)$. Denote by $x_{i}(t)=x_{i}\left(t ; 0, \phi_{i}\right), x_{i} \in \Omega, i=1,2$ the solution of system

(2) satisfying the initial conditions

$$
0 \leq x_{i}\left(s ; 0, \phi_{i}\right)=\phi_{i}(s)<+\infty, \quad s \in[-\tau, 0], \quad x_{i}\left(0+0 ; 0, \phi_{i}\right)=\phi_{i}(0)>0 .
$$

By the basic theories of impulsive differential equations with delay in Stamov (2012), system (2) and (5) has a unique solution. Since the solution of system (2) and (5) is a piecewise continuous function with points of discontinuity of the first kind $\tau_{k}, k \in \mathbb{Z}$ we adopt the following definitions for almost periodicity.

Definition 1 (Stamov 2012) The integer number $p$ is said to be an $\epsilon$-almost period of $\left\{\tau_{k}\right\}$, if for $k \in \mathbb{Z},\left|\tau_{k+p}-\tau_{k}\right|<\epsilon$. 
Definition 2 (Stamov 2012) The set of sequences $\left\{\tau_{k}^{j}\right\}, \tau_{k}^{j}=\tau_{k+j}-\tau_{k}$, $k \in \mathbb{Z}, j \in \mathbb{Z},\left\{\tau_{k}\right\} \in \mathbb{I}$ is said to be uniformly almost periodic if for arbitrary $\epsilon>0$ there exists a relatively dense set of $\epsilon$-almost periods common for any sequences.

Definition 3 (Stamov 2012) The function $\varphi \in P C(\mathbb{R}, \mathbb{R})$ is said to be almost periodic, if the following hold:

(1) The set of sequences $\left\{\tau_{k}^{j}\right\}, \tau_{k}^{j}=\tau_{k+j}-\tau_{k}, k \in \mathbb{Z}, j \in \mathbb{Z},\left\{\tau_{k}\right\} \in \mathbb{I}$ is uniformly almost periodic.

(2) For any $\epsilon>0$ there exists a real number $\delta>0$ such that if the points $t^{\prime}$ and $t^{\prime \prime}$ belong to one and the same interval of continuity of $\varphi(t)$ and satisfy the inequality $\left|t^{\prime}-t^{\prime \prime}\right|<\delta$, then $\left|\varphi\left(t^{\prime}\right)-\varphi\left(t^{\prime \prime}\right)\right|<\epsilon$.

(3) For any $\epsilon>0$ there exists a relatively dense set $T$ such that if $\eta \in T$, then $|\varphi(t+\eta)-\varphi(t)|<\epsilon$ for all $t \in \mathbb{R}$ satisfying the condition $\left|t-\tau_{k}\right|>\epsilon, k \in \mathbb{Z}$. The elements of $T$ are called $\epsilon$-almost periods.

Lemma 1 (Stamov 2012) Let $\left\{\tau_{k}\right\} \in \mathbb{I}$ Then there exists a positive integer. A such that on each interval of length 1 , we have no more than $A$ elements of the sequence $\left\{\tau_{k}\right\}$, i.e.,

$$
i(s, t) \leq A(t-s)+A \text {, }
$$

where $i(s, t)$ is the number of the points $\tau_{k}$ in the interval $(s, t)$.

Theoretically, one can investigate the existence, uniqueness and stability of almost periodic solution for functional differential equations by using Lyapunov functional as follows (Stamov 2012, $\mathrm{P}_{109}$ ):

Let $\mathbb{R}^{n}$ be the $n$-dimensional Euclidean space with elements $x=\left(x_{1}, \ldots, x_{n}\right)^{T}$ and norm $|x|_{0}=\sum_{i=1}^{n}\left|x_{i}\right|, C=C\left([-\tau, 0], \mathbb{R}^{n}\right), \mathbb{B} \in \mathbb{R}^{+}$. Denote $C_{\mathbb{B}}=\{\varphi \in C:\|\varphi\|<\mathbb{B}\}$, with $\|\varphi\|=\sup _{s \in[-\tau, 0]}|\varphi(s)|_{0}$.

Consider the system of impulsive differential equations with delay:

$$
\left\{\begin{array}{l}
\dot{x}(t)=f\left(t, x_{t}\right), \quad t \neq \tau_{k}, \\
\Delta x\left(\tau_{k}\right)=I_{k}\left(x\left(\tau_{k}\right)\right),
\end{array}\right.
$$

where $t \in \mathbb{R},\left\{\tau_{k}\right\} \in \mathbb{I}, f(t, \varphi)$ is continuous in $(t, \varphi) \in \mathbb{R} \times C_{\mathbb{B}}$ and almost periodic in $t$ uniformly for $\varphi \in C_{\mathbb{B}}, \forall \rho>0, \exists M(\rho)>0$ such that $|f(t, \varphi)| \leq M(\rho)$ as $t \in \mathbb{R}, \varphi \in C_{\rho}$, while $x_{t} \in C_{\mathbb{B}}$ is defined as $x_{t}(s)=x(t+s)$ for $s \in[-\tau, 0], I_{k}: D \rightarrow \mathbb{R}^{n}, k \in \mathbb{Z}, D$ is an open set in $\mathbb{R}^{n}$.

Introduce the following conditions:

$\left(C_{1}\right)$ The sequence $\left\{I_{k}(x)\right\}, k \in \mathbb{Z}$ is almost periodic uniformly with respect to $x \in D$.

Lemma 2 (Stamov 2012, $\mathrm{P}_{109}$ ) Suppose that there exists a Lyapunov functional $V(t, \phi, \psi)$ defined on $\mathbb{R} \times C_{\mathbb{B}} \times C_{\mathbb{B}}$ satisfying the following conditions:

(1) $u(\|\phi-\psi\|) \leq V(t, \phi, \psi) \leq v(\|\phi-\psi\|)$, where $u, v \in \mathcal{P}$ with $\mathcal{P}=\left\{u: \mathbb{R}^{+} \rightarrow \mathbb{R}^{+} \mid u\right.$ is continuous increasing function and $u(s) \rightarrow 0$ as $s \rightarrow 0\}$. 
(2) $|V(t, \bar{\phi}, \bar{\psi})-V(t, \hat{\phi}, \hat{\psi})| \leq \mathrm{L}(\|\bar{\phi}-\hat{\phi}\|+\|\bar{\psi}-\hat{\psi}\|)$, where $\mathrm{L}>0$ is a constant.

(3) For $t=\tau_{k}, V\left(t^{+}, \phi+I_{k}(\phi), \psi+I_{k}(\psi)\right) \leq V(t, \phi, \psi)$; For $t \neq \tau_{k}, \dot{V}_{(2.2)}(t, \phi, \psi) \leq$ $-\gamma V(t, \phi, \psi), \forall k \in \mathbb{Z}$, where $\gamma>0$ is a constant.

Moreover, one assumes that system (6) has a solution that remains in a compact set $S \subset D$. Then system (6) has a unique almost periodic solution which is uniformly asymptotically stable.

Remark 2 From the proof of Lemma 2, it is not difficult to prove that condition (1) of Lemma 2 can be replaced by the following condition:

$(1)^{\prime} u\left(|\phi(0)-\psi(0)|_{0}\right) \leq V(t, \phi, \psi) \leq v(\|\phi-\psi\|)$, where $u, v \in \mathcal{P}$ with $\mathcal{P}=\left\{u: \mathbb{R}^{+}\right.$

$\rightarrow \mathbb{R}^{+} \mid u$ is continuous increasing function and $u(s) \rightarrow 0$ as $\left.s \rightarrow 0\right\}$.

\section{Permanence}

In this section, we establish a permanence result for system (2).

Lemma 3 (Lakshmikantham et al. 1989) Assume that $x \in P C(\mathbb{R})$ with points of discontinuity at $t=\tau_{k}$ and is left continuous at $t=\tau_{k}$ for $k \in \mathbb{Z}^{+}$, and

$$
\begin{cases}\dot{x}(t) \leq f(t, x(t)), & t \neq \tau_{k}, \\ x\left(\tau_{k}^{+}\right) \leq I_{k}\left(x\left(\tau_{k}\right)\right), & k \in \mathbb{Z}^{+},\end{cases}
$$

where $f \in C(\mathbb{R} \times \mathbb{R}, \mathbb{R}), I_{k} \in C(\mathbb{R}, \mathbb{R})$ and $I_{k}(x)$ is nondecreasing in $x$ for $k \in \mathbb{Z}^{+}$. Let $u^{*}(t)$ be the maximal solution of the scalar impulsive differential equation

$$
\begin{cases}\dot{u}(t)=f(t, u(t)), & t \neq \tau_{k}, \\ u\left(\tau_{k}^{+}\right)=I_{k}\left(u\left(\tau_{k}\right)\right) \geq 0, & k \in \mathbb{Z}^{+}, \\ u\left(t_{0}^{+}\right)=u_{0} & \end{cases}
$$

existing on $\left[t_{0}, \infty\right)$. Then $x\left(t_{0}^{+}\right) \leq u_{0}$ implies $x(t) \leq u^{*}(t)$ for $t \geq t_{0}$.

Remark 3 If the inequalities (7) in Lemma 3 is reversed and $u_{*}(t)$ is the minimal solution of system (8) existing on $\left[t_{0}, \infty\right)$, then $x\left(t_{0}^{+}\right) \geq u_{0}$ implies $x(t) \geq u_{*}(t)$ for $t \geq t_{0}$.

For arbitrary $a, b>0, h_{k} \geq 0$, we give the following notations:

$$
\begin{aligned}
\xi: & =\ln _{k \in \mathbb{Z}} \frac{1}{1+h_{k}}, \quad \alpha:=a-\xi A, \quad \theta:=\inf _{k \in \mathbb{Z}} \tau_{k}^{1}, \quad \eta:=\inf _{k \in \mathbb{Z}}\left\{\prod_{j=0}^{1} \frac{1}{1+h_{j+k}}, 1\right\}, \\
W(t, s) & = \begin{cases}e^{-a(t-s)}, & \tau_{k-1}<s<t<\tau_{k} ; \\
\prod_{j=m}^{k+1} \frac{1}{1+h_{j}} e^{-a(t-s)}, & \tau_{m-1}<s \leq \tau_{m}<\tau_{k}<t \leq \tau_{k+1},\end{cases}
\end{aligned}
$$

where $A$ is defined as that in Lemma $1, \tau_{k}^{1}=\tau_{k+1}-\tau_{k}$ is defined as that in Definition 2 .

Lemma 4 Assume that $a, b>0, h_{k} \geq 0$, then the following impulsive logistic equation

$$
\left\{\begin{array}{l}
\dot{x}(t)=x(t)[a-b x(t)], \quad t \neq \tau_{k}, \\
\Delta x\left(\tau_{k}\right)=h_{k} x\left(\tau_{k}\right), \quad k \in \mathbb{Z}^{+}
\end{array}\right.
$$


has a unique globally asymptotically stable positive almost periodic solution $x^{*}$, which can be expressed as follows:

$$
\frac{\alpha}{e^{\xi A} b} \leq x^{*}(t)=\left[b \int_{-\infty}^{t} W(t, s) \mathrm{d} s\right]^{-1} \leq \frac{a}{\eta b\left(1-e^{-a \theta}\right)}
$$

Proof Let $u=\frac{1}{x}$, then system (9) changes to

$$
\begin{cases}\frac{\mathrm{d} u(t)}{\mathrm{d} t}=-a u(t)+b, & t \neq \tau_{k} \\ \Delta u\left(\tau_{k}\right)=-\frac{h_{k}}{1+h_{k}} u\left(\tau_{k}\right), & k \in \mathbb{Z}^{+} .\end{cases}
$$

Together with the system (11) we consider the linear system

$$
\begin{cases}\frac{\mathrm{d} u(t)}{\mathrm{d} t}=-a u(t), & t \neq \tau_{k}, \\ \Delta u\left(\tau_{k}\right)=-\frac{h_{k}}{1+h_{k}} u\left(\tau_{k}\right), & k \in \mathbb{Z}^{+} .\end{cases}
$$

Now let us consider the equation

$$
\frac{\mathrm{d} u(t)}{\mathrm{d} t}=-a u(t), \quad \tau_{k-1}<t \leq \tau_{k}
$$

and its solution

$$
u(t)=u(s) e^{-a(t-s)}, \quad \tau_{k-1}<s<t \leq \tau_{k} .
$$

Then from Stamov (2012), the Cauchy matrix of the linear system (12) is

$$
W(t, s)= \begin{cases}e^{-a(t-s)}, & \tau_{k-1}<s<t<\tau_{k} \\ \prod_{j=m}^{k+1} \frac{1}{1+h_{j}} e^{-a(t-s)}, & \tau_{m-1}<s \leq \tau_{m}<\tau_{k}<t \leq \tau_{k+1}\end{cases}
$$

and the solution of system (12) is in the form

$$
u\left(t ; t_{0} ; u\left(t_{0}\right)\right)=W\left(t, t_{0}\right) u\left(t_{0}\right), \quad t_{0} \in \mathbb{R} .
$$

Therefore, system (11) has a solution

$$
u\left(t ; t_{0} ; u\left(t_{0}\right)\right)=W\left(t, t_{0}\right) u\left(t_{0}\right)+b \int_{t_{0}}^{t} W(t, s) \mathrm{d} s .
$$

Letting $t_{0} \rightarrow-\infty$ in the above equation $\left(W\left(t, t_{0}\right) u\left(t_{0}\right) \rightarrow 0\right)$, then by Stamov (2012) we have

$$
u(t)=b \int_{-\infty}^{t} W(t, s) \mathrm{d} s
$$

is a solution of system (11) and is almost periodic. Then system (9) has a almost periodic solution $x^{*}(t)$ which can be expressed by (10). By Lemma 1 , we have from (10) that

$$
x^{*}(t) \geq\left[b \int_{-\infty}^{t} e^{i(s, t) \xi} e^{-a(t-s)} \mathrm{d} s\right]^{-1} \geq\left[b \int_{-\infty}^{t} e^{\xi A} e^{-\alpha(t-s)} \mathrm{d} s\right]^{-1}=\frac{\alpha}{e^{\xi A} b}
$$


On the other hand,

$$
x^{*}(t) \leq\left[b \int_{t-\theta}^{t} W(t, s) \mathrm{d} s\right]^{-1} \leq\left[b \int_{t-\theta}^{t} \eta e^{-a(t-s)} \mathrm{d} s\right]^{-1}=\frac{a}{\eta b\left(1-e^{-a \theta}\right)} .
$$

Next, we shall prove that the uniqueness and stability of $x^{*}(t)$ of system (9). Suppose that $x(t)$ is another positive solution of system (9). Define a function

$$
V(t)=\left|\ln x^{*}(t)-\ln x(t)\right|, \quad \forall t \in \mathbb{R} .
$$

For $t \neq \tau_{k}, k \in \mathbb{Z}^{+}$, calculating the upper right derivative of $V(t)$ along the solution of system (9), we have

$$
D^{+} V(t)=\operatorname{sgn}\left[x^{*}(t)-x(t)\right]\left[\frac{\dot{x}^{*}(t)}{x^{*}(t)}-\frac{\dot{x}(t)}{x(t)}\right]=-b\left|x^{*}(t)-x(t)\right| .
$$

For $t=\tau_{k}, k \in \mathbb{Z}^{+}$, we have

$$
\begin{aligned}
V\left(\tau_{k}^{+}\right) & =\left|\ln x^{*}\left(\tau_{k}^{+}\right)-\ln x\left(\tau_{k}^{+}\right)\right| \\
& =\left|\ln \frac{\left(1+h_{k}\right) x^{*}\left(\tau_{k}\right)}{\left(1+h_{k}\right) x\left(\tau_{k}\right)}\right|=\left|\ln x^{*}\left(\tau_{k}\right)-\ln x\left(\tau_{k}\right)\right|=V\left(\tau_{k}\right) .
\end{aligned}
$$

Therefore, $V$ is non-increasing. Integrating (13) from 0 to $t$ leads to

$$
V(t)+b \int_{0}^{t}\left|x(s)-x^{*}(s)\right| \mathrm{d} s \leq V(0)<+\infty, \quad \forall t \geq 0,
$$

that is,

$$
\int_{0}^{+\infty}\left|x(s)-x^{*}(s)\right| \mathrm{d} s<+\infty
$$

which implies that

$$
\lim _{s \rightarrow+\infty}\left|x(s)-x^{*}(s)\right|=0
$$

Thus, the almost periodic solution of system (9) is globally asymptotically stable. This completes the proof.

Lemma 5 Assume that $a, b>0, h_{k} \geq 0$, then every solution $x$ of the following system with delay

$$
\left\{\begin{array}{l}
\dot{x}(t) \leq x(t)[a-b x(t-\tau)], \quad t \neq \tau_{k}, \\
\Delta x\left(\tau_{k}\right)=h_{k} x\left(\tau_{k}\right), \quad k \in \mathbb{Z}^{+}
\end{array}\right.
$$

satisfies

$$
\lim \sup _{t \rightarrow \infty} x(t) \leq M:=\frac{a}{\eta B\left(1-e^{-a \theta}\right)},
$$

where $B=\inf _{t \in \mathbb{R}} b \prod_{\tau_{k} \in[t-\tau, t)}\left(1+h_{k}\right)^{-1} e^{-a \tau}, \theta:=\inf _{k \in \mathbb{Z}} \tau_{k}^{1}$ and $\eta:=\inf _{k \in \mathbb{Z}} \prod_{j=1}^{2} \frac{1}{1+h_{j+k}}$. 
Proof From system (14), we have

$$
\left\{\begin{array}{l}
\dot{x}(t) \leq a x(t), \quad t \neq \tau_{k}, \\
\Delta x\left(\tau_{k}\right)=h_{k} x\left(\tau_{k}\right), \quad k \in \mathbb{Z}^{+}
\end{array}\right.
$$

is equivalent to

$$
\left\{\begin{array}{l}
\frac{\mathrm{d}}{\mathrm{dt}}\left[x(t) e^{-a t}\right] \leq 0, \quad t \neq \tau_{k}, \\
\Delta x\left(\tau_{k}\right)=h_{k} x\left(\tau_{k}\right), \quad k \in \mathbb{Z}^{+} .
\end{array}\right.
$$

For some $t \in[0,+\infty)$ and $t \neq \tau_{k}, k \in \mathbb{Z}^{+}$, consider interval $[t-\tau, t)$. Assume that $\tau_{1}<\tau_{2}<\cdots<\tau_{j}$ are the impulse points in $[t-\tau, t)$. Integrating the first inequality of system (15) from $t-\tau$ to $\tau_{1}$ leads to

$$
x\left(\tau_{1}\right) e^{-a \tau_{1}} \leq x(t-\tau) e^{-a(t-\tau)} .
$$

Integrating the first inequality of system (15) from $\tau_{1}$ to $\tau_{2}$ leads to

$$
x\left(\tau_{2}\right) e^{-a \tau_{2}} \leq x\left(\tau_{1}^{+}\right) e^{-a \tau_{1}}=\left(1+h_{1}\right) x\left(\tau_{1}\right) e^{-a \tau_{1}} \leq\left(1+h_{1}\right) x(t-\tau) e^{-a(t-\tau)} .
$$

Integrating the first inequality of system (15) from $\tau_{2}$ to $\tau_{3}$ leads to

$$
x\left(\tau_{3}\right) e^{-a \tau_{3}} \leq x\left(\tau_{2}^{+}\right) e^{-a \tau_{2}}=\left(1+h_{2}\right) x\left(\tau_{2}\right) e^{-a \tau_{2}} \leq\left(1+h_{1}\right)\left(1+h_{2}\right) x(t-\tau) e^{-a(t-\tau)} .
$$

Repeating the above process, integrating the first inequality of system (15) from $\tau_{j}$ to $t$ leads to

$$
x(t) e^{-a t} \leq x\left(\tau_{j}^{+}\right) e^{-a \tau_{j}}=\left(1+h_{j}\right) x\left(\tau_{j}\right) e^{-a \tau_{j}} \leq \prod_{\tau_{k} \in[t-\tau, t)}\left(1+h_{k}\right) x(t-\tau) e^{-a(t-\tau)} .
$$

Then

$$
x(t-\tau) \geq \prod_{\tau_{k} \in[t-\tau, t)}\left(1+h_{k}\right)^{-1} e^{-a \tau} x(t) .
$$

Substituting (16) into system (14) leads to

$$
\left\{\begin{array}{l}
\dot{x}(t) \leq x(t)[a-B x(t)], \quad t \neq \tau_{k}, \\
\Delta x\left(\tau_{k}\right)=h_{k} x\left(\tau_{k}\right), \quad k \in \mathbb{Z}^{+} .
\end{array}\right.
$$

Consider the auxiliary system

$$
\begin{cases}\dot{z}(t)=z(t)[a-B z(t)], & t \neq \tau_{k}, \\ z\left(\tau_{k}^{+}\right)=\left(1+h_{k}\right) z\left(\tau_{k}\right), & k \in \mathbb{Z}^{+} .\end{cases}
$$

By Lemma 3, $x(t) \leq z(t)$, where $z(t)$ is the solution of system (17) with $z\left(0^{+}\right)=x\left(0^{+}\right)$. By Lemma 4 , system (17) has a unique globally asymptotically stable positive almost periodic solution $z^{*}$ which can be expressed as follows:

$$
z^{*}(t)=\left[B \int_{-\infty}^{t} W(t, s) \mathrm{d} s\right]^{-1} \leq\left[B \int_{t-\theta}^{t} W(t, s) \mathrm{d} s\right]^{-1} \leq \frac{a}{\eta B\left(1-e^{-a \theta}\right)}:=M .
$$


Then for any constant $\epsilon>0$, there exists $T_{1}>0$ such that $x(t) \leq z(t)<z^{*}(t)+$ $\epsilon \leq M+\epsilon$ for $t>T_{1}$. So

$$
\lim \sup _{t \rightarrow \infty} x(t) \leq M
$$

This completes the proof.

Lemma 6 Assume that $a, b>0, h_{k} \geq 0$, then every solution $x$ of the following system with delay

$$
\left\{\begin{array}{l}
\dot{x}(t) \geq x(t)[a-b x(t-\tau)], \quad t \neq \tau_{k}, \\
\Delta x\left(\tau_{k}\right)=h_{k} x\left(\tau_{k}\right), \quad k \in \mathbb{Z}^{+}
\end{array}\right.
$$

satisfies

$$
\lim \inf _{t \rightarrow \infty} x(t) \geq N:=\frac{a-\xi A}{e^{\xi A} D},
$$

where $\quad D=\sup _{t \in \mathbb{R}} b \prod_{\tau_{k} \in[t-\tau, t)}\left(1+h_{k}\right)^{-1} e^{-(a-b M) \tau}, \quad \xi:=\ln \sup _{k \in \mathbb{Z}} \frac{1}{1+h_{k}}$ and $A$ is defined as that in Lemma 1.

Proof According to Lemma 5, there exist $\epsilon>0$ and $T_{2}>0$ such that

$$
x(t) \leq M+\epsilon \quad \text { for } t \geq T_{2} .
$$

From system (18), we have

$$
\left\{\begin{array}{l}
\dot{x}(t) \geq[a-b(M+\epsilon)] x(t), \quad t \neq \tau_{k}, \quad t \geq T_{2}, \\
\Delta x\left(\tau_{k}\right)=h_{k} x\left(\tau_{k}\right), \quad k \in \mathbb{Z}^{+}
\end{array}\right.
$$

is equivalent to

$$
\left\{\begin{array}{l}
\frac{\mathrm{d}}{\mathrm{dt}}\left[x(t) e^{-[a-b(M+\epsilon)] t}\right] \geq 0, \quad t \neq \tau_{k} \quad t \geq T_{2}, \\
\Delta x\left(\tau_{k}\right)=h_{k} x\left(\tau_{k}\right), \quad k \in \mathbb{Z}^{+} .
\end{array}\right.
$$

Similar to the argument as that in (16), we have

$$
b x(t-\tau) \leq b \prod_{\tau_{k} \in[t-\tau, t)}\left(1+h_{k}\right)^{-1} e^{-[a-b(M+\epsilon)] \tau} x(t):=D_{\epsilon} x(t), \quad t \geq T_{2} .
$$

Substituting (19) into system (18) leads to

$$
\left\{\begin{array}{l}
\dot{x}(t) \geq x(t)\left[a-D_{\epsilon} x(t)\right], \quad t \neq \tau_{k}, \quad t \geq T_{2}, \\
\Delta x\left(\tau_{k}\right)=h_{k} x\left(\tau_{k}\right), \quad k \in \mathbb{Z}^{+} .
\end{array}\right.
$$

Consider the auxiliary system

$$
\left\{\begin{array}{l}
\dot{z}(t)=z(t)\left[a-D_{\epsilon} z(t)\right], \quad t \neq \tau_{k}, \quad t \geq T_{2}, \\
z\left(\tau_{k}^{+}\right)=\left(1+h_{k}\right) z\left(\tau_{k}\right), \quad k \in \mathbb{Z}^{+} .
\end{array}\right.
$$


By Remark 3, $x(t) \geq z(t)$, where $z(t)$ is the solution of system (20) with $z\left(0^{+}\right)=x\left(0^{+}\right)$. By Lemma 4 , system (20) has a unique globally asymptotically stable positive almost periodic solution $z^{*}$ which can be expressed as follows:

$$
z^{*}(t)=\left[D_{\epsilon} \int_{-\infty}^{t} W(t, s) \mathrm{d} s\right]^{-1} \geq\left[D_{\epsilon} \int_{t-\theta}^{t} W(t, s) \mathrm{d} s\right]^{-1} \geq \frac{a-\xi A}{e^{\xi A} D_{\epsilon}}
$$

Letting $\epsilon \rightarrow 0$ in the above inequality leads to

$$
z^{*}(t) \geq \frac{a-\xi A}{e^{\xi A} D}:=N
$$

Similar to the argument as that in Lemma 5, it follows that

$$
\lim \inf _{t \rightarrow \infty} x(t) \geq N
$$

This completes the proof.

Remark 4 When $h_{i k}(i=1,2) \equiv 0$ in systems (14) and (18), then Lemmas 5, 6 change to the corresponding results in Nakata and Muroya (2010). So Lemmas 5, 6 extend the corresponding result in Nakata and Muroya (2010).

Let

$$
\eta_{i}:=\inf _{k \in \mathbb{Z}} \prod_{j=0}^{1} \frac{1}{1+h_{i(j+k)}}, \quad \xi_{i}:=\ln \sup _{k \in \mathbb{Z}} \frac{1}{1+h_{i k}}, \quad i=1,2 .
$$

Proposition 1 Every solution $\left(x_{1}, x_{2}\right)^{T}$ of system (2) satisfies

$$
\lim \sup _{t \rightarrow \infty} x_{i}(t) \leq M_{i}, \quad i=1,2,
$$

where $M_{1}$ and $M_{2}$ are defined as that in (21) and (22), respectively.

Proof From system (1), we have

$$
\left\{\begin{array}{l}
\dot{x}_{1}(t) \leq x_{1}(t)\left[r_{1}^{u}-a_{11}^{l} x_{1}\left(t-\tau_{11}\right)\right], \quad t \neq \tau_{k} \\
x_{1}\left(\tau_{k}^{+}\right)=\left(1+h_{1 k}\right) x_{1}\left(\tau_{k}\right), \quad k \in \mathbb{Z}^{+}
\end{array}\right.
$$

where $r_{1}^{u}:=\sup _{t \in \mathbb{R}}\left|\frac{a_{10}(t)}{m_{1}(t)}-c_{1}(t)\right|$. By Lemma 5, we have

$$
\lim \sup _{t \rightarrow \infty} x_{1}(t) \leq M_{1}:=\frac{r_{1}^{u}}{\eta_{1} B_{1}\left(1-e^{-r_{1}^{u} \theta}\right)},
$$

where $B_{1}=\inf _{t \in \mathbb{R}} a_{11}^{l} \prod_{\tau_{k} \in\left[t-\tau_{11}, t\right)}\left(1+h_{1 k}\right)^{-1} e^{-r_{1}^{u} \tau_{11}}$. Similarly, ones obtain that

$$
\lim \sup _{t \rightarrow \infty} x_{2}(t) \leq M_{2}:=\frac{r_{2}^{u}}{\eta_{2} B_{2}\left(1-e^{-r_{2}^{u} \theta}\right)},
$$


where $r_{2}^{u}:=\sup _{t \in \mathbb{R}}\left|\frac{a_{20}(t)}{m_{2}(t)}-c_{2}(t)\right|, B_{2}=\inf _{t \in \mathbb{R}} a_{22}^{l} \prod_{\tau_{k} \in\left[t-\tau_{22}, t\right)}\left(1+h_{2 k}\right)^{-1} e^{-r_{2}^{u} \tau_{22}}$. This completes the proof.

Proposition 2 Let $N_{1}$ and $N_{2}$ are defined as that in (23) and (24), respectively. Then every solution $\left(x_{1}, x_{2}\right)^{T}$ of system (2) satisfies

$$
\lim \inf _{t \rightarrow \infty} x_{i}(t) \geq N_{i}, \quad i=1,2,
$$

if the following condition holds:

$\begin{aligned}\left(H_{1}\right) \quad r_{1}^{l} & :=\inf _{t \in \mathbb{R}}\left[\frac{a_{10}(t)}{M_{1}+m_{1}(t)}-a_{12}(t) M_{2}-c_{1}(t)\right] \geq \xi_{1} A \\ r_{2}^{l} & :=\inf _{t \in \mathbb{R}}\left[\frac{a_{20}(t)}{M_{2}+m_{2}(t)}-a_{21}(t) M_{1}-c_{2}(t)\right] \geq \xi_{2} A .\end{aligned}$

Proof According to Proposition 1 and $\left(H_{1}\right)$, there exist $\epsilon>0$ and $T_{3}>0$ such that

$$
\begin{aligned}
& x_{i}(t) \leq M_{i}+\epsilon \quad \text { for } t \geq T_{3}, \quad i=1,2, \\
& r_{1}^{l}(\epsilon):=\inf _{t \in \mathbb{R}}\left[\frac{a_{10}(t)}{\left(M_{1}+\epsilon\right)+m_{1}(t)}-a_{12}(t)\left(M_{2}+\epsilon\right)-c_{1}(t)\right] \geq \xi_{1} A, \\
& r_{2}^{l}(\epsilon):=\inf _{t \in \mathbb{R}}\left[\frac{a_{20}(t)}{\left(M_{2}+\epsilon\right)+m_{2}(t)}-a_{21}(t)\left(M_{1}+\epsilon\right)-c_{2}(t)\right] \geq \xi_{2} A .
\end{aligned}
$$

From system (1), we have

$$
\left\{\begin{array}{l}
\dot{x}_{1}(t) \geq x_{1}(t)\left[r_{1}^{l}(\epsilon)-a_{11}^{u} x_{1}\left(t-\tau_{11}\right)\right], \quad t \neq \tau_{k}, t>T_{3}, \\
x_{1}\left(\tau_{k}^{+}\right)=\left(1+h_{1 k}\right) x_{1}\left(\tau_{k}\right), \quad k \in \mathbb{Z}^{+} .
\end{array}\right.
$$

By Lemma 6 and the arbitrariness of $\epsilon$, one has

$$
\lim \inf _{t \rightarrow \infty} x_{1}(t) \geq N_{1}:=\frac{r_{1}^{l}-\xi_{1} A}{e^{\xi_{1} A} D_{1}}
$$

where $D_{1}:=\sup _{t \in \mathbb{R}} a_{11}^{u} \prod_{\tau_{k} \in\left[t-\tau_{11}, t\right)}\left(1+h_{1 k}\right)^{-1} e^{-\left(r_{1}^{l}-a_{11}^{u} M_{1}\right) \tau_{11}}$. Similarly, we have

$$
\lim \inf _{t \rightarrow \infty} x_{2}(t) \geq N_{2}:=\frac{r_{2}^{l}-\xi_{2} A}{e^{\xi_{2} A} D_{2}}
$$

where $\quad D_{2}:=\sup _{t \in \mathbb{R}} a_{22}^{u} \prod_{\tau_{k} \in\left[t-\tau_{22}, t\right)}\left(1+h_{2 k}\right)^{-1} e^{-\left(r_{2}^{l}-a_{22}^{u} M_{2}\right) \tau_{22}}$. This completes the proof.

Remark 5 In view of $\left(H_{1}\right)$ in Proposition 2, the values of impulse coefficients $h_{i k}(i=1,2)$ and the number of the impulse points $\tau_{k}$ in each interval of length 1 have negative effect on the permanence of system (2).

By Propositions 1, 2, we have

Theorem 1 Assume that $\left(H_{1}\right)$ holds, then system (2) is permanent.

Remark 6 Theorem 1 is a permanence result of system (2) without $(F)$. So Theorem 1 improves the corresponding result in Zhang et al. (2012). Further, Theorem 1 provides 
a possible and effective method to study the permanence of the models with impulsive perturbations and pure-delays in biological populations.

Remark 7 From the proof of Propositions 1, 2, we know that under the conditions of Theorem 1, the set $S=\left\{\left(x_{1}, x_{2}\right)^{T} \in \mathbb{R}^{2}: N_{i} \leq x_{i} \leq M_{i}, i=1,2\right\}$ is an invariant set of system (2).

\section{Almost periodic solution}

The main result of this paper concerns the existence of a unique uniformly asymptotically stable positive almost periodic solution for system (2).

For convenience, we introduce some notations as follows:

$$
\begin{aligned}
& \alpha_{1}=\frac{a_{10}^{l} N_{1}}{\left(M_{1}+m_{1}^{u}\right)^{2}}+a_{11}^{l} N_{1}-\left(\frac{\sqrt{\tau_{10}} a_{10}^{u} M_{1}}{\left(N_{1}+m_{1}^{l}\right)^{2}}\right)^{2}-\frac{\left(\tau_{10}+\tau_{11}\right) a_{10}^{u} a_{11}^{u} M_{1}^{2}}{\left(N_{1}+m_{1}^{l}\right)^{2}}-\tau_{11} a_{11}^{u 2} M_{1}^{2}, \\
& \alpha_{2}=\frac{a_{20}^{l} N_{2}}{\left(M_{2}+m_{2}^{u}\right)^{2}}+a_{22}^{l} N_{2}-\left[\frac{\sqrt{\tau_{20}} a_{20}^{u} M_{2}}{\left(N_{2}+m_{2}^{l}\right)^{2}}\right]^{2}-\frac{\left(\tau_{20}+\tau_{22}\right) a_{20}^{u} a_{22}^{u} M_{2}^{2}}{\left(N_{2}+m_{2}^{l}\right)^{2}}-\tau_{22} a_{22}^{u 2} M_{2}^{2}, \\
& \beta_{1}=\frac{\tau_{20} a_{20}^{u} a_{21}^{u} M_{1} M_{2}}{\left(N_{2}+m_{2}^{l}\right)^{2}}+\tau_{22} a_{21}^{u} a_{22}^{u} M_{1} M_{2}+a_{21}^{u} M_{1}, \\
& \beta_{2}=\frac{\tau_{10} a_{10}^{u} a_{12}^{u} M_{1} M_{2}}{\left(N_{1}+m_{1}^{l}\right)^{2}}+\tau_{11} a_{11}^{u} a_{12}^{u} M_{1} M_{2}+a_{12}^{u} M_{2},
\end{aligned}
$$

where $M_{1}, M_{2}, N_{1}$ and $N_{2}$ are defined as that in "Permanence" section.

\section{Theorem 2 Assume that $\left(H_{1}\right)$ holds. Suppose further that}

$\left(H_{2}\right)$ There exist two positive constants $\lambda_{1}$ and $\lambda_{2}$ such that $\lambda_{1} \alpha_{1}>\lambda_{2} \beta_{1}$ and $\lambda_{2} \alpha_{2}>\lambda_{1} \beta_{2}$. Then system (2) admits a unique uniformly asymptotically stable almost periodic solution.

Proof Let $x_{i}(t)=e^{z_{i}(t)}, i=1,2$, then system (2) is transformed into

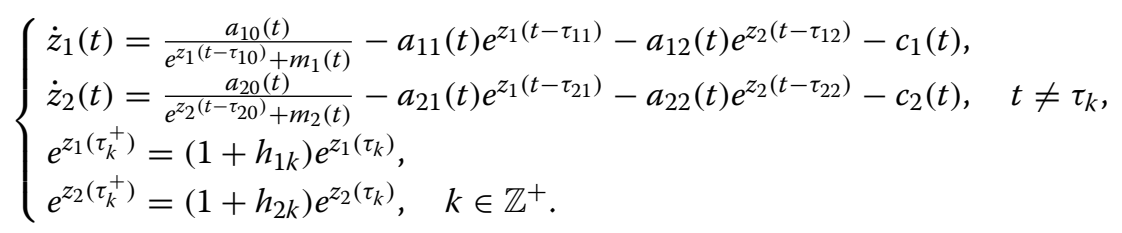

Suppose that $Z(t)=\left(z_{1}(t), z_{2}(t)\right)^{T}$ and $Z^{*}(t)=\left(z_{1}^{*}(t), z_{2}^{*}(t)\right)^{T}$ are any two solutions of system (25). Consider the product system of system (25)

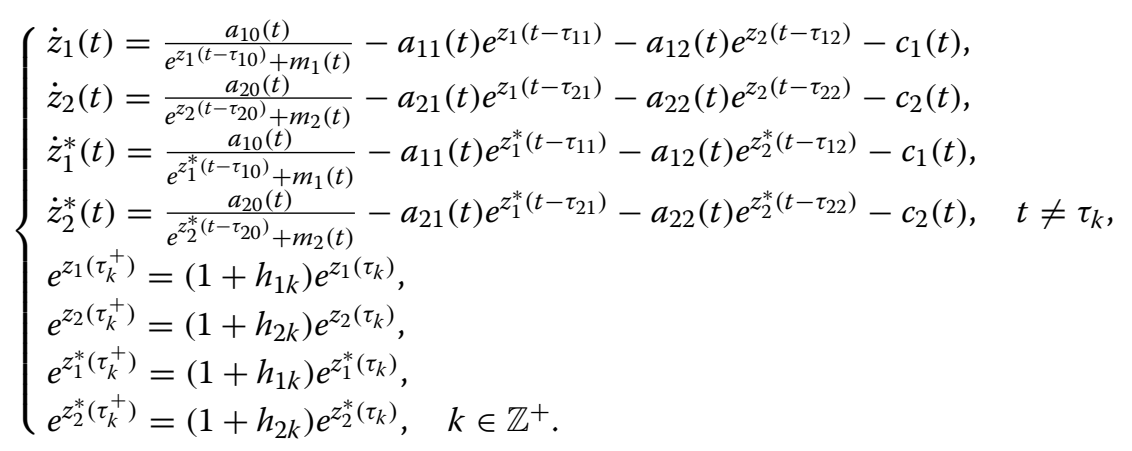


Set $S_{1}=\left\{\phi=\left(z_{1 t}, z_{2 t}\right)^{T} \in C\left([-\tau, 0], \mathbb{R}^{2}\right): \ln N_{i} \leq z_{i t} \leq \ln M_{i}, t \in \mathbb{R}^{+}, i=1,2\right\}$, which is an invariant set of system (26) directly from Remark 7 .

Construct a Lyapunov functional $V(t)=V(t, \phi, \psi)=V\left(t,\left(z_{1 t}, z_{2 t}\right)^{T},\left(z_{1 t}^{*}, z_{2 t}^{*}\right)^{T}\right)$ defined on $\mathbb{R}^{+} \times S_{1} \times S_{1}$ as follows:

$V(t, \phi, \psi)=V_{1}(t, \phi, \psi)+V_{2}(t, \phi, \psi)+V_{3}(t, \phi, \psi)+V_{4}(t, \phi, \psi)$,

where

$$
\begin{aligned}
V_{1}(t, \phi, \psi)= & \lambda_{1}\left|z_{1}(t)-z_{1}^{*}(t)\right|+\lambda_{2}\left|z_{2}(t)-z_{2}^{*}(t)\right|, \\
V_{2}(t, \phi, \psi)= & \lambda_{1}\left(\frac{a_{10}^{u} M_{1}}{\left(N_{1}+m_{1}^{l}\right)^{2}}\right)^{2} \int_{-2 \tau_{10}}^{-\tau_{10}} \int_{t+s}^{t}\left|z_{1}(r)-z_{1}^{*}(r)\right| \mathrm{d} r \mathrm{~d} s \\
& +\lambda_{1} \frac{a_{10}^{u} a_{11}^{u} M_{1}^{2}}{\left(N_{1}+m_{1}^{l}\right)^{2}} \int_{-\tau_{11}-\tau_{10}}^{-\tau_{11}} \int_{t+s}^{t}\left|z_{1}(r)-z_{1}^{*}(r)\right| \mathrm{d} r \mathrm{~d} s \\
& +\lambda_{1} \frac{a_{10}^{u} a_{11}^{u} M_{1}^{2}}{\left(N_{1}+m_{1}^{l}\right)^{2}} \int_{-\tau_{10}-\tau_{11}}^{-\tau_{10}} \int_{t+s}^{t}\left|z_{1}(r)-z_{1}^{*}(r)\right| \mathrm{d} r \mathrm{~d} s \\
& +\lambda_{1} a_{11}^{u 2} M_{1}^{2} \int_{-2 \tau_{11}}^{-\tau_{11}} \int_{t+s}^{t}\left|z_{1}(r)-z_{1}^{*}(r)\right| \mathrm{d} r \mathrm{~d} s \\
& +\lambda_{2} \frac{a_{20}^{u} a_{21}^{u} M_{1} M_{2}}{\left(N_{2}+m_{2}^{l}\right)^{2}} \int_{-\tau_{21}-\tau_{20}}^{-\tau_{21}} \int_{t+s}^{t}\left|z_{1}(r)-z_{1}^{*}(r)\right| \mathrm{d} r \mathrm{~d} s \\
& +\lambda_{2} a_{21}^{u} a_{22}^{u} M_{1} M_{2} \int_{-\tau_{21}-\tau_{22}}^{-\tau_{21}} \int_{t+s}^{t}\left|z_{1}(r)-z_{1}^{*}(r)\right| \mathrm{d} r \mathrm{~d} s, \\
V_{3}(t, \phi, \psi)= & \lambda_{1} \frac{a_{10}^{u} a_{12}^{u} M_{1} M_{2}}{\left(N_{1}+m_{1}^{l}\right)^{2}} \int_{-\tau_{12}-\tau_{10}}^{-\tau_{12}} \int_{t+s}^{t}\left|z_{2}(r)-z_{2}^{*}(r)\right| \mathrm{d} r \mathrm{~d} s \\
& +\lambda_{1} a_{11}^{u} a_{12}^{u} M_{1} M_{2} \int_{-\tau_{12}-\tau_{11}}^{-\tau_{12}} \int_{t+s}^{t}\left|z_{2}(r)-z_{2}^{*}(r)\right| \mathrm{d} r \mathrm{~d} s \\
& +\lambda_{2}\left(\frac{a_{20}^{u} M_{2}}{\left(N_{2}+m_{2}^{l}\right)^{2}}\right)^{2} \int_{-2 \tau_{20}}^{-\tau_{20}} \int_{t+s}^{t}\left|z_{2}(r)-z_{2}^{*}(r)\right| \mathrm{d} r \mathrm{~d} s \\
& +\lambda_{2} \frac{a_{20}^{u} a_{22}^{u} M_{2}^{2}}{\left(N_{2}+m_{2}^{l}\right)^{2}} \int_{-\tau_{22}-\tau_{20}}^{-\tau_{22}} \int_{t+s}^{t}\left|z_{2}(r)-z_{2}^{*}(r)\right| \mathrm{d} r \mathrm{~d} s \\
& +\lambda_{2} \frac{a_{20}^{u} a_{22}^{u} M_{2}^{2}}{\left(N_{2}+m_{2}^{l}\right)^{2}} \int_{-\tau_{20}-\tau_{22}}^{-\tau_{20}} \int_{t+s}^{t}\left|z_{2}(r)-z_{2}^{*}(r)\right| \mathrm{d} r \mathrm{~d} s \\
V_{4}(t, \phi, \psi)= & \lambda_{2} a_{21}^{u} M_{1} \int_{t-\tau_{21}}^{t}\left|z_{1}(s)-z_{1}^{*}(s)\right| \mathrm{d} s+\lambda_{1} a_{12}^{u} M_{2} \int_{t-\tau_{12}}^{t}\left|z_{2}(s)-z_{2}^{*}(s)\right| \mathrm{d} s . \\
t+s & z_{2}^{*}(r) \mid \mathrm{d} r \mathrm{~d} s, \\
& \\
& \\
&
\end{aligned}
$$

By the definitions of $S_{1}$ and $V$, there is some large enough positive constant $K$ such that

$$
V(t, \phi, \psi) \leq K .
$$

Similar to the argument as that in Zhang et al. (2012), we have

$$
V(t, \phi, \psi) \geq \underline{\lambda}|\phi(0)-\psi(0)|_{0},
$$

where $\underline{\lambda}:=\min \left\{\lambda_{1}, \lambda_{2}\right\}$ 


$$
V(t, \phi, \psi) \leq \bar{\lambda}\|\phi-\psi\|,
$$

where

$$
\begin{aligned}
\bar{\lambda}:= & \lambda_{1}+\lambda_{2}+2 \lambda_{1} \tau^{2}\left(\frac{a_{10}^{u} M_{1}}{\left(N_{1}+m_{1}^{l}\right)^{2}}\right)^{2}+2 \lambda_{1} \tau^{2} \frac{a_{10}^{u} a_{11}^{u} M_{1}^{2}}{\left(N_{1}+m_{1}^{l}\right)^{2}} \\
& +2 \lambda_{1} \tau^{2} \frac{a_{10}^{u} a_{11}^{u} M_{1}^{2}}{\left(N_{1}+m_{1}^{l}\right)^{2}}+2 \lambda_{1} \tau^{2} a_{11}^{u 2} M_{1}^{2}+2 \lambda_{2} \tau^{2} \frac{a_{20}^{u} a_{21}^{u} M_{1} M_{2}}{\left(N_{2}+m_{2}^{l}\right)^{2}} \\
& +2 \lambda_{2} \tau^{2} a_{21}^{u} a_{22}^{u} M_{1} M_{2}+2 \lambda_{1} \tau^{2} \frac{a_{10}^{u} a_{12}^{u} M_{1} M_{2}}{\left(N_{1}+m_{1}^{l}\right)^{2}}+2 \lambda_{1} \tau^{2} a_{11}^{u} a_{12}^{u} M_{1} M_{2} \\
& +2 \lambda_{2} \tau^{2}\left(\frac{a_{20}^{u} M_{2}}{\left(N_{2}+m_{2}^{l}\right)^{2}}\right)^{2}+2 \lambda_{2} \tau^{2} \frac{a_{20}^{u} a_{22}^{u} M_{2}}{\left(N_{2}+m_{2}^{l}\right)^{2}}+2 \lambda_{2} \tau^{2} \frac{a_{20}^{u} a_{22}^{u} M_{2}^{2}}{\left(N_{2}+m_{2}^{l}\right)^{2}} \\
& +2 \lambda_{2} \tau^{2} a_{22}^{u 2} M_{2}^{2}+\lambda_{2} \tau a_{21}^{u} M_{1}+\lambda_{1} \tau a_{12}^{u} M_{2},
\end{aligned}
$$

and for $\forall \bar{\phi}=\left(\bar{z}_{1 t}, \bar{z}_{2 t}\right)^{T}, \bar{\psi}=\left(\bar{z}_{1 t}^{*}, \bar{z}_{2 t}^{*}\right)^{T}, \hat{\phi}=\left(\hat{z}_{1 t}, \hat{z}_{2 t}\right)^{T}, \hat{\psi}=\left(\hat{z}_{1 t}^{*}, \hat{z}_{2 t}^{*}\right)^{T} \in S_{1}$, it follows that

$$
|V(t, \bar{\phi}, \bar{\psi})-V(t, \hat{\phi}, \hat{\psi})| \leq \bar{\lambda}(\|\bar{\phi}-\hat{\phi}\|+\|\bar{\psi}-\hat{\psi}\|) .
$$

So condition (2) in Lemma 2 is satisfied. In view of (27)-(28), let $u, v \in C\left(\mathbb{R}^{+}, \mathbb{R}^{+}\right)$, $u(s)=\underline{\lambda} s, v(s)=\bar{\lambda} s$, thus condition (1) $)^{\prime}$ in Remark 2 is satisfied.

From article Zhang et al. (2012), for $t \neq \tau_{k}, k \in \mathbb{Z}^{+}$, calculating the upper right derivative of $V$ along the solution of system (26), we have

$$
D^{+} V(t, \phi, \psi) \leq-\gamma V(t, \phi, \psi)
$$

where $\gamma:=\frac{\chi \lambda|\phi(0)-\psi(0)|_{0}}{K}, \chi:=\min \left\{\frac{\Theta}{\lambda_{1}}, \frac{\Theta}{\lambda_{2}}\right\}$ and $\Theta:=\min \left\{\lambda_{1} \alpha_{1}-\lambda_{2} \beta_{1}, \lambda_{2} \alpha_{2}-\lambda_{1} \beta_{2}\right\}$.

For $t=\tau_{k}, k \in \mathbb{Z}^{+}$, we have

$$
\begin{aligned}
V\left(\tau_{k}^{+}, \phi, \psi\right) & =V_{1}\left(\tau_{k}^{+}, \phi, \psi\right)+V_{2}\left(\tau_{k}^{+}, \phi, \psi\right)+V_{3}\left(\tau_{k}^{+}, \phi, \psi\right)+V_{4}\left(\tau_{k}^{+}, \phi, \psi\right) \\
& =V_{1}\left(\tau_{k}^{+}, \phi, \psi\right)+V_{2}\left(\tau_{k}, \phi, \psi\right)+V_{3}\left(\tau_{k}, \phi, \psi\right)+V_{4}\left(\tau_{k}, \phi, \psi\right) \\
& =\sum_{i=1}^{2} \lambda_{i}\left|z_{i}\left(\tau_{k}^{+}\right)-z_{i}^{*}\left(\tau_{k}^{+}\right)\right|+V_{2}\left(\tau_{k}, \phi, \psi\right)+V_{3}\left(\tau_{k}, \phi, \psi\right)+V_{4}\left(\tau_{k}, \phi, \psi\right) \\
& =\sum_{i=1}^{2} \lambda_{i}\left|z_{i}\left(\tau_{k}\right)-z_{i}^{*}\left(\tau_{k}\right)\right|+V_{2}\left(\tau_{k}, \phi, \psi\right)+V_{3}\left(\tau_{k}, \phi, \psi\right)+V_{4}\left(\tau_{k}, \phi, \psi\right) \\
& =V_{1}\left(\tau_{k}, \phi, \psi\right)+V_{2}\left(\tau_{k}, \phi, \psi\right)+V_{3}\left(\tau_{k}, \phi, \psi\right)+V_{4}\left(\tau_{k}, \phi, \psi\right) \\
& =V\left(\tau_{k}, \phi, \psi\right) .
\end{aligned}
$$

In view of (29)-(30), condition (3) in Lemma 2 is satisfied.

By Lemma 2, system (2) admits a unique uniformly asymptotically stable positive almost periodic solution $\left(z_{1}(t), z_{2}(t)\right)^{T}$. This completes the proof.

Remark 8 Without $(F)$, system (2) also admits a unique uniformly asymptotically stable positive almost periodic solution. So Theorem 2 extends the corresponding result 
in Zhang et al. (2012). Further, Theorem 2 gives the sufficient conditions for the uniform asymptotical stability of a unique positive almost periodic solution of system (2). Therefore, Theorem 2 provides a possible method to study the existence, uniqueness and stability of positive almost periodic solution of the models with impulsive perturbations and pure-delays in biological populations.

Remark 9 In the last two decades, the method of constructing a Lyapunov functional has been extensively used in the study of stability of the deterministic models (Xue et al. 2015; Tian et al. 2015; Zhang et al. 2015; Liu and Xu 2004; Liu et al. 2006; Li and Yang 2009; Gan and Lin 2012; Wu et al. 2009; Zhang et al. 2012). However, there have been numerous relevant works using the Lyapunov functional method in stochastic systems, see Shang $(2014,2015,2016)$. The methods used in this paper can be extended to study the permanence and existence of a unique uniformly asymptotically stable positive almost periodic solution of the stochastic models with impulsive perturbations in biological populations.

\section{An example and numerical simulations}

Example 1 Consider the following Schoener's competition model with pure-delays and impulsive effects:

$$
\left\{\begin{array}{l}
\dot{x}_{1}(t)=x_{1}(t)\left\{\frac{1}{x_{1}(t-0.0001)+2}-a_{11}(t) x_{1}(t-0.0001)-a_{12}(t) x_{2}(t-0.0002)-c_{1}(t)\right\}, \\
\dot{x}_{2}(t)=x_{2}(t)\left\{\frac{1}{x_{2}(t-0.0001)+2}-a_{21}(t) x_{1}(t-0.0002)-a_{22}(t) x_{2}(t-0.0001)-c_{2}(t)\right\}, \quad t \neq \tau_{k}, \\
\Delta x_{1}\left(\tau_{k}\right)=0.5 x_{1}\left(\tau_{k}\right), \\
\Delta x_{2}\left(\tau_{k}\right)=0.4 x_{2}\left(\tau_{k}\right), \quad\left\{\tau_{k}: k \in \mathbb{Z}\right\} \subset\{5 k: k \in \mathbb{Z}\},
\end{array}\right.
$$

where $\quad a_{11}(t)=a_{22}(t)=0.35+0.05 \cos (\sqrt{3} t), \quad a_{12}(t)=a_{21}(t) \equiv 0.0001, \quad c_{1}(t)=$ $0.30005+0.00005 \sin (\sqrt{2} t), c_{2}(t)=0.30005+0.00005 \cos (\sqrt{2} t), t \in \mathbb{R}$. Then the above system is permanent and has a unique uniformly asymptotically stable almost periodic solution.

Proof Obviously, $a_{10}^{u}=a_{20}^{u}=1, \quad a_{10}^{l}=a_{20}^{l}=1, \quad a_{11}^{u}=a_{22}^{u}=0.4, \quad a_{11}^{l}=a_{22}^{l}=0.3$, $a_{12}^{u}=a_{21}^{u}=0.0001, \quad a_{12}^{l}=a_{21}^{l}=0.0001, \quad c_{1}^{u}=c_{2}^{u}=0.3001, \quad c_{1}^{l}=c_{2}^{l}=0.3, \quad \theta=2$, $\eta_{1} \approx 0.4, \eta_{2}=4, \xi_{1} \approx 0.3, \xi_{2} \approx 0.7, A=1$. By calculation, we obtain that $y_{1}^{*} \approx 0.7256$, $y_{2}^{*} \approx 0.5421, \min \left\{r_{1}^{l}, r_{2}^{l}\right\} \geq 0.03>0, y_{1 *}=y_{2 *} \geq 0.01$. So $\left(H_{1}\right)$ holds. Further, we also get that

$$
\alpha_{1} \geq 0.03, \quad \alpha_{2} \geq 0.02, \quad \max \left\{\beta_{1}, \beta_{2}\right\} \leq 0.00007,
$$

which implies that $\left(H_{2}\right)$ is satisfied for $\lambda_{1}=\lambda_{2}=1$. It is easy to verify that $\left(H_{1}\right)-\left(H_{2}\right)$ are satisfied and the result follows from Theorems 1 and 2 (see Figs. 1, 2, 3). This completes the proof. 


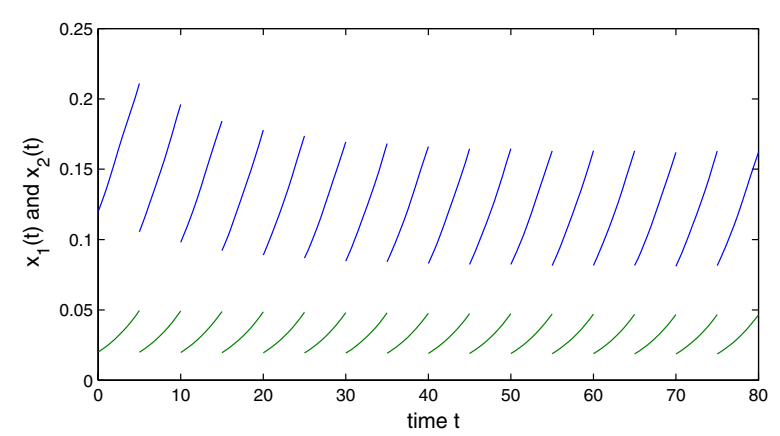

Fig. 1 State variables $x_{1}$ and $x_{2}$ of Example 1. Blue line $x_{1}(s)=0.12, s \in[-1,0]$; Green line $x_{2}(s)=0.02$, $s \in[-1,0]$

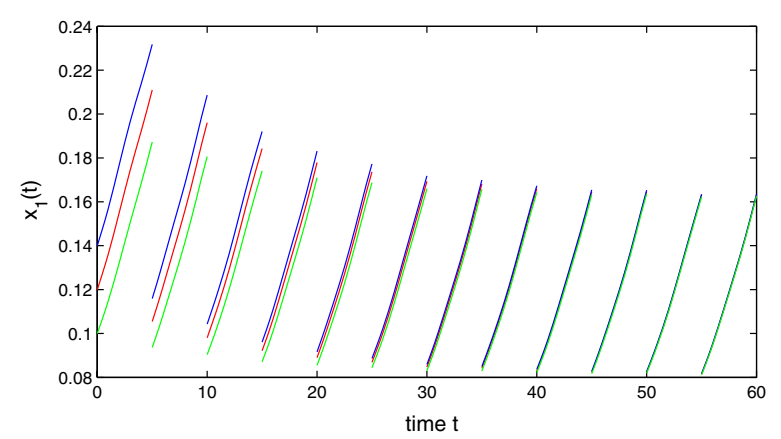

Fig. 2 Stability of state variable $x_{1}$ of Example 1. Blue line $x_{1}(s)=0.14, s \in[-1,0]$; Red line $x_{1}(s)=0.12$, $s \in[-1,0]$; Green line $x_{1}(s)=0.10, s \in[-1,0]$

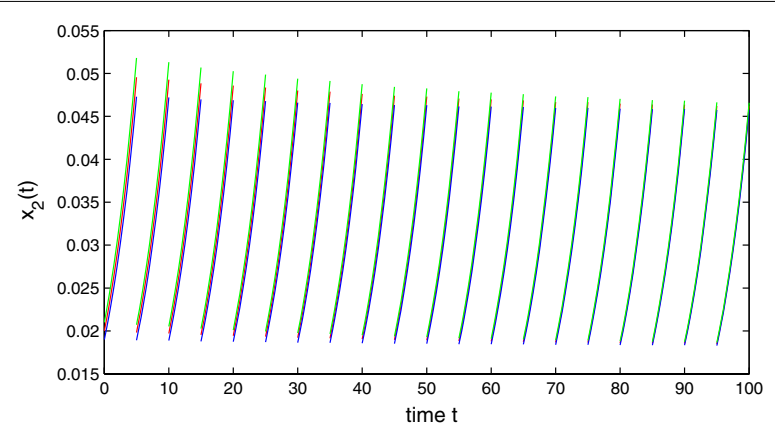

Fig. 3 Stability of state variable $x_{2}$ of Example 1. Blue line $x_{2}(s)=0.018, s \in[-1,0]$; Red line $x_{2}(s)=0.002$, $s \in[-1,0]$; Green line $x_{1}(s)=0.022, s \in[-1,0]$

Remark 10 In Example 1, the impulse coefficients $\left(h_{1 k}=0.5\right.$ and $\left.h_{2 k}=0.6\right)$ do not satisfy $(F)$. So it is impossible to obtain the permanence and existence of a unique uniformly asymptotically stable positive almost periodic solution of system (1) by the result in Zhang et al. (2012). Therefore, the work in this paper improves the results in paper Zhang et al. (2012).

\section{Conclusion}

By using the comparison theorem and the Lyapunov method of the impulsive differential equations, sufficient conditions are obtained for the permanence and existence of 
a unique uniformly asymptotically stable positive almost periodic solution in a class of impulsive Schoener's competition model with pure-delays. Proposition 2 and Theorem 2 imply that the values of impulse coefficients $h_{i k}(i=1,2,3)$ and the number of the impulse points $\tau_{k}$ in each interval of length 1 are harm for the permanence and existence of a unique uniformly asymptotically stable positive almost periodic solution for a class of impulsive Schoener's competition model with pure-delays. The main results obtained in this paper are completely new and the method used in this paper provides a possible method to study the permanence and existence of a unique uniformly asymptotically stable positive almost periodic solution of the models with impulsive perturbations and pure-delays in biological populations.

Authors' contributions

CL, ZGG and ZYZ collectively carried out this study, collected data, and analyzed them. CL and ZGG wrote the manuscript. ZYZ helped to draft the manuscript. All authors read and approved the final manuscript.

\section{Acknowledgements}

This work are supported by the Natural Science Foundation of Shanxi Province under Grant 2011011002-3, the Project of University Educational Reform in Shanxi Province under Grant J2015118 and the Education Science Foundation of Shanxi Province under Grant GH-13118. The authors thank the referee for his/her careful reading of the original manuscript and valuable comments and suggestions that greatly improved the presentation of this work.

\section{Competing interests}

The authors declare that they have no competing interests.

Received: 13 November 2015 Accepted: 29 March 2016

Published online: 14 April 2016

\section{References}

Bainov DD, Simeonov PS (1993) Impulsive differential equations: periodic solutions and applications. Longman Scientific and Technical, New York

Chen LS (1988) Mathematical ecology modelling and research methods. Science Press, Beijing (in Chinese)

Du ZJ, Lv YS (2013) Permanence and almost periodic solution of a Lotka-Volterra model with mutual interference and time delays. Appl Math Model 37:1054-1068

Gan WZ, Lin ZG (2012) The asymptotic periodicity in a Schoener's competitive model. Appl Math Model 36:989-996

He MX, Chen FD, Li Z (2010) Almost periodic solution of an impulsive differential equation model of plankton allelopathy. Nonlinear Anal Real World Appl 11:2296-2301

Jin Z, Han MA, Li GH (2005) The persistence in a Lotka-Volterra competition systems with impulsive. Chaos Solitons Fractals 24:1105-1117

Lakshmikantham V, Bainov DD, Simeonov PS (1989) Theory of impulsive differential equations. World Scientific, Singapore

Li XP, Yang WS (2009) Permanence of a discrete $n$-species schoener competition system with time delays and feedback controls. Adv Differ Equ 2009:1-10

Liao YZ, Zhang TW (2012) Almost periodic solutions of a discrete mutualism model with variable delays. Discrete Dyn Nat Soc 2012:1-27

Liao YZ, Xu LJ (2014) Almost periodic solution for a delayed Lotka-Volterra system on time scales. Adv Differ Equ 2014:1-19

Lin X, Chen FD (2009) Almost periodic solution for a Volterra model with mutual interference and Beddington-DeAngelis functional response. Appl Math Comput 214:548-556

Liu ZJ, Chen LS (2007) Periodic solution of a two-species competitive system with toxicant and birth pulse. Chaos Solitons Fractals 32:1703-1712

Liu QM, Xu R (2004) Periodic solutions of Schoener's competitive model with delays. J Biomath 19:385-394 (in Chinese)

Liu QM, Xu R, Wang WG (2006) Global asymptotic stability of Schoener's competitive model with time delays. J Biomath 21:147-152

Liu QM, Xu R, Wang WG (2006) Global asymptotic stability of Schoener's competitive model with delays. J Biomath 21:147-152 (in Chinese)

Meng XZ, Chen LS (2006) Almost periodic solution of non-autonomous Lotka-Volterra predator-prey dispersal system with delays. J Theor Biol 243:562-574

Nakata Y, Muroya Y (2010) Permanence for nonautonomous Lotka-Volterra cooperative systems with delays. Nonlinear Anal Real World Appl 11:528-534

Samoilenko AM, Perestyuk NA (1995) Impulsive differential equations. World Scientific, Singapore

Shang YL (2014) Group pinning consensus under fixed and randomly switching topologies with acyclic partition. Netw Heterog Media 9:553-573

Shang YL (2015) Global stability of disease-free equilibria in a two-group SI model with feedback control. Nonlinear Anal Model Control 20:501-508 
Shang YL (2016) Consensus seeking over Markovian switching networks with time-varying delays and uncertain topologies. Appl Math Comput 273:1234-1245

Stamov GT (2009) On the existence of almost periodic solutions for the impulsive Lasota-Wazewska model. Appl Math Lett 22:516-520

Stamov GT (2012) Almost periodic solutions of impulsive differential equations. In: Lecture notes in mathematics. Springer, Berlin

Tian BD, Chen N, Qiu YH (2015) Almost periodic solution for an epidemic prey-predator system with impulsive effects and multiple delays. Discrete Dyn Nat Soc 2015:1-15

Wu LP, Chen FD, Li Z (2009) Permanence and global attractivity of a discrete Schoener's competition model with delays. Math Comput Model 49:1607-1617

Xia YH, Han MA, Huang ZK (2008) Global attractivity of an almost periodic N-species nonlinear ecological competitive model. J Math Anal Appl 337:144-168

Xia YH (2013) Theory and applications of periodic solutions and almost periodic solutions. Discrete Dyn Nat Soc 2013:1-2

Xue YL, Xie XD, Chen FD, Han RY (2015) Almost periodic solution of a discrete commensalism system. Discrete Dyn Nat Soc 2015:1-11

Zhang TW (2013) Multiplicity of positive almost periodic solutions in a delayed Hassell-Varleytype predator-prey model with harvesting on prey. Math Methods Appl Sci 37:686-697

Zhang TW (2014) Almost periodic oscillations in a generalized Mackey-Glass model of respiratory dynamics with several delays. Int J Biomath 7:1-22

Zhang TW, Li YK (2011) Positive periodic solutions for a generalized impulsive $n$-species Gilpin-Ayala competition system with continuously distributed delays on time scales. Int J Biomath 4:23-34

Zhang H, Li YQ, Jing B (2014) Global attractivity and almost periodic solution of a discrete mutualism model with delays. Math Methods Appl Sci 37:3013-3025

Zhang H, Li YQ, Jing B, Zhao WZ (2014) Global stability of almost periodic solution of multispecies mutualism system with time delays and impulsive effects. Appl Math Comput 232:1138-1150

Zhang TW, Li YK, Ye Y (2012) On the existence and stability of a unique almost periodic solution of Schoener's competition model with pure-delays and impulsive effects. Commun Nonlinear Sci Numer Simul 17:1408-1422

Zhang H, Feng F, Jing B, Li YQ (2015) Almost periodic solution of a multispecies discrete mutualism system with feedback controls. Discrete Dyn Nat Soc 2015:1-14

Zhou H, Wang J, Zhou Z (2013) Positive almost periodic solution for impulsive Nicholson's blowflies model with multiple linear harvesting terms. Math Methods Appl Sci 36:456-461

\section{Submit your manuscript to a SpringerOpen ${ }^{\circ}$ journal and benefit from:}

- Convenient online submission

- Rigorous peer review

- Immediate publication on acceptance

- Open access: articles freely available online

- High visibility within the field

- Retaining the copyright to your article

Submit your next manuscript at $\mathbf{s p r i n g e r o p e n . c o m ~}$ 